\title{
In vitro Investigation of the Antimicrobial Activity of a Series of Lipophilic Phenols and Naphthols
}

\author{
Thavendran Govender, ${ }^{\text {a }}$ Usha Govinden, ${ }^{\text {a }}$ Chunderika Mocktar, ${ }^{\text {a }}$ Hendrik G. Kruger, ${ }^{a}$ \\ Jelena Veljković, ${ }^{\mathrm{b}}$ Nikola Cindro, ${ }^{\mathrm{b}}$ Damir Bobinac, ${ }^{\mathrm{b}}$ Ivana Žabčić, ${ }^{\mathrm{b}}$ \\ Kata Mlinarić-Majerski ${ }^{b}$ and Nikola Basaric ${ }^{b_{*}}$ \\ ${ }^{a}$ Catalysis and Peptide Research Unit, School of Health Sciences, University of KwaZulu-Natal, Durban, South Africa. \\ ${ }^{b}$ Department of Organic Chemistry and Biochemistry, Ruđer Bošković Institute, Bijenička cesta 54, 10000 Zagreb, Croatia.
}

Received 11 August 2015, revised 20 January 2016, accepted 5 February 2016.

\begin{abstract}
Five groups of phenols/naphthols (42 compounds in total) were synthesized and screened against Gram-positive Staphylococcus aureus and Bacillus subtilis, Gram-negative Escherichia coli and Klebsiella pneumoniae, and the fungus Candida albicans. Whereas compounds were found inactive against Gram-negative bacteria, potent activities against Gram-positive bacteria were observed. The activities correlate with the ability of molecules to form quinone methides, suggesting potential new modes of action.
\end{abstract}

KEYWORDS

Antimicrobial activity, phenols, naphthols, quinone methides.

\section{Introduction}

Bacterial infections are responsible for a vast number of human diseases. Moreover, development of bacterial resistence to common antibiotics is stimulating intensive research devoted to discovery of new targets in combating the bacteria. In search for new and improved antibiotics with new mechanism of action, it is important to screen many new libraries of compounds.

Antimicrobial activity of simple phenols, ${ }^{1}$ and their carboxylic acid derivatives has been investigated. ${ }^{2}$ It is generally accepted that phenols and benzyl alcohols exert antimicrobial action ${ }^{3-6}$ on the non-specific ability to alter membranes in Gram-negative bacteria. ${ }^{7-10}$ On the other hand, a more specific mode of action of phenolic compounds has been suggested, especially against Gram-positive bacteria, since the bactericidal concentrations are not dependent on the type of cell wall. ${ }^{11}$ It was suggested that phenolic compounds inhibit DNA synthesis, due to an effect associated with the inhibition of RNA and protein synthesis. ${ }^{11}$ Furthermore, antibacterial properties are ascribed to a large number of food products due to the presence of phenolic natural compounds. ${ }^{12}$ Examples include berries ${ }_{1}^{13}$ vine ${ }_{1}^{14}$ tropical vegetables, ${ }^{15}$ and mushrooms. ${ }^{16}$ Recently, an investigation of antimicrobial activities have been conducted on a series of naphthol derivatives of Mannich bases. ${ }^{17}$ The latter series of compounds can in principle be transformed to quinone methides (QM) intermediates during metabolic processes. QMs are interesting substrates in

* To whom correspondence should be addressed. E-mail: nbasaric@irb.hr $\mathrm{R} \rightarrow$<smiles>[CH2]c1ccc(CC(C)(Cl)Cl)cc1</smiles>
E-Ser-OH
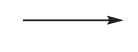

E-Ser-OCOR

the development of antimicrobial agents since it was demonstrated that they act as alkylating agents and inhibitors of serine proteases and $\beta$-lactamases. ${ }^{18}$ Depending on their structure, they are mechanism-based enzyme inhibitors, or suicide substrates. When QMs are formed from cyclic compounds such as coumarins, they usually induce cross-linking of the enzyme in the reaction with a histidine. On the contrary, free QM formed in the active site of the enzyme usually reacts with neighbouring tryptophan residues (Scheme 1). ${ }^{18}$

We became interested in the photochemical generation of QMs with sterically congested lipophylic substituents ${ }^{19,20}$ and their antiproliferative activities. ${ }^{21-24}$ A series of simple phenols, ${ }^{19}$ biphenyls, ${ }^{20-22}$ naphthols, ${ }^{23}$ and anthrols ${ }^{24}$ were synthesized and their antiproliferative activities were investigated. Since compounds that generate QMs are known $\beta$-lactamase inhibitors, ${ }^{18}$ we were also prompted to investigate their antimicrobic activities. Potentially, they can be used in combination with $\beta$-lactam derivatives as antibiotics. Herein we report an investigation of the antimicrobial activity of five series of lipophilic derivatives of phenols and naphthols and/or benzyl alcohols (Figs. 1-5) with the aim of developing a SAR relationship. Moreover, many of our derivatives bear an adamantyl substituent, and the antibacterial activity of a number of adamantane derivatives has been reported. ${ }^{25-29}$ The in vitro antimicrobial testing was performed on five strains of microorganisms, Gram-positive Staphylococcus aureus and Bacillus subtilis, Gram-negative Escherichia coli and Klebsiella pneumoniae, and the fungus Candida albicans.

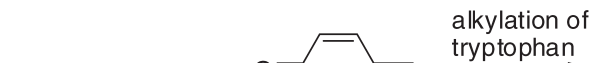
\begin{tabular}{l} 
tryptophan \\
\hline
\end{tabular}

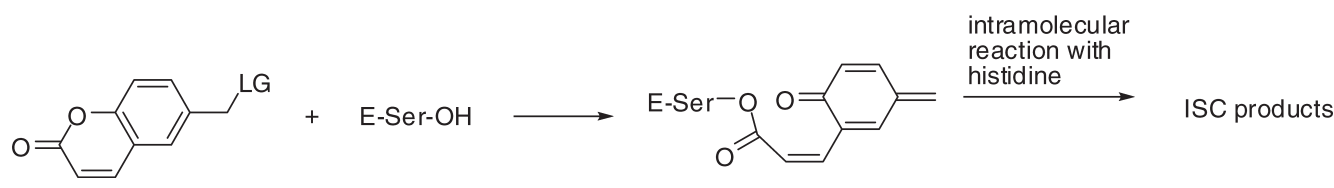

Scheme 1 


\section{Matherials and Methods}

\subsection{Compounds}

The structures of the investigated compounds comprise phenols (Fig. 1), 2-hydroxybiphenyls (Fig. 2), 3-hydroxybiphenyls (Fig. 3), 4-hydroxybiphenyls (Fig. 4), naphthols (Fig. 5) or their corresponding methoxy or QM derivatives. All compounds were prepared according to previously published procedures. ${ }^{19-23}$ Chiral compounds 7-9 were synthesized as racemic mixtures.

\subsubsection{Synthesis of Phenol Derivatives 1-9}

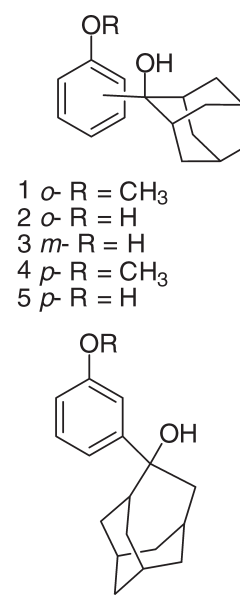

$( \pm)-7 \mathrm{R}=\mathrm{CH}_{3}$

$( \pm)-8 \mathrm{R}=\mathrm{H}$

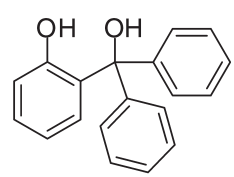

6

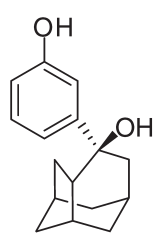

$( \pm)-9$
Figure 1 Phenol derivatives.

2-Hydroxy-2-(2-methoxyphenyl)adamantane (1) was prepared in a Grignard reaction from 2-adamantanone and 2-bromanisol in $50 \%$ yield according to the published procedure. ${ }^{19}$ Characterization of product 1 compares well with literature $(\mathrm{mp}=$ $\left.87-88^{\circ} \mathrm{C}\right) .{ }^{19}{ }^{1} \mathrm{H}$ NMR $\left(\mathrm{CDCl}_{3}, 300 \mathrm{MHz}\right) \delta / \mathrm{ppm}: 7.44(\mathrm{dd}, 1 \mathrm{H}, J=$ $1.5 \mathrm{~Hz}, J=7.7 \mathrm{~Hz}), 7.21(\mathrm{dt}, 1 \mathrm{H}, J=1.5 \mathrm{~Hz}, J=8.1 \mathrm{~Hz}), 6.93(\mathrm{dt}, 1 \mathrm{H}$, $J=1.5 \mathrm{~Hz}, J=7.7 \mathrm{~Hz}), 6.90(\mathrm{~d}, 1 \mathrm{H}, J=8.1 \mathrm{~Hz}), 3.85(\mathrm{~s}, 3 \mathrm{H}), 3.42(\mathrm{~s}$, $1 \mathrm{H}, \mathrm{OH}), 2.66$ (br s, 2H), 2.54 (br s, 1H), 2.50 (br s, 1H), 1.65-1.90 $(\mathrm{m}, 8 \mathrm{H}), 1.61(\mathrm{~d}, J=12.5 \mathrm{~Hz}, 2 \mathrm{H})$.

2-Hydroxy-2-(2-hydroxyphenyl)adamantane (2) was prepared from 1 by treatment with $\mathrm{BBr}_{3}$ in $45 \%$ yield according to the published procedure. ${ }^{19}$ Characterization of product 2 compares well with literature $\left(\mathrm{mp}=170-171^{\circ} \mathrm{C}\right) \cdot{ }^{19} \mathrm{H}$ NMR (DMSO- $d_{6}, 300$

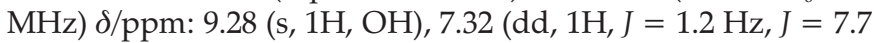
$\mathrm{Hz}), 7.05(\mathrm{dt}, 1 \mathrm{H}, J=1.5 \mathrm{~Hz}, J=8.5 \mathrm{~Hz}), 6.73-6.82(\mathrm{~m}, 2 \mathrm{H}) 4.93(\mathrm{~s}$, $1 \mathrm{H}, \mathrm{OH}), 2.61$ (br s, 2H), 2.44 (br s, 1H), 2.40 (br s, 1H), 1.60-1.50 $(\mathrm{m}, 8 \mathrm{H}), 1.51(\mathrm{~d}, 2 \mathrm{H}, \mathrm{J}=12 \mathrm{~Hz})$.

2-Hydroxy-2-(3-hydroxyphenyl)adamantane (3) was prepared from 2-hydroxy-2-(3-methoxyphenyl)adamantane by treatment with $\mathrm{BBr}_{3}$ in $85 \%$ yield according to the published procedure. ${ }^{19}$ Characterization of product 3 compares well with literature $\left(\mathrm{mp}=160-161^{\circ} \mathrm{C}\right) .{ }^{19}{ }^{1} \mathrm{H}$ NMR (DMSO- $\left.d_{6}, 600 \mathrm{MHz}\right) \delta / \mathrm{ppm}: 9.17$ $(\mathrm{s}, 1 \mathrm{H}, \mathrm{OH}), 7.11(\mathrm{t}, 1 \mathrm{H}, J=7.7 \mathrm{~Hz}), 6.87-6.90(\mathrm{~m}, 2 \mathrm{H}), 6.60(\mathrm{ddd}$, $1 \mathrm{H}, J=0.9 \mathrm{~Hz}, J=2.3 \mathrm{~Hz}, J=7.7 \mathrm{~Hz}), 4.48(\mathrm{~s}, 1 \mathrm{H}, \mathrm{OH}), 2.37(\mathrm{br} \mathrm{s}$, $3 \mathrm{H}), 2.35$ (br s, 1H), 1.79 (br s, 1H), 1.52-1.68 (m, 9H).

2-Hydroxy-2-(4-methoxyphenyl)adamantane (4) was prepared in a Grignard reaction from 2-adamantanone and 4-bromanisol in $65 \%$ yield according to the published procedure. ${ }^{19}$ Characterization of product 4 compares well with literature $(\mathrm{mp}=$ 104-105 $\left.{ }^{\circ} \mathrm{C}\right) .{ }^{19}{ }^{1} \mathrm{H}$ NMR $\left(\mathrm{CDCl}_{3}, 300 \mathrm{MHz}\right) \delta /$ ppm: $7.46(\mathrm{~d}, 2 \mathrm{H}$,
$J=8.8 \mathrm{~Hz}), 6.89(\mathrm{~d}, 2 \mathrm{H}, J=8.8 \mathrm{~Hz}), 3.80\left(\mathrm{~s}, 3 \mathrm{H}, \mathrm{OCH}_{3}\right), 2.52(\mathrm{br} \mathrm{s}$, $2 \mathrm{H}), 2.42$ (br s, 1H), 2.38 (br s, 1H), 1.89 (br s, 1H), 1.65-1.75 (m, 9H), 1.49 (br s, $1 \mathrm{H}, \mathrm{OH})$.

2-Hydroxy-2-(4-hydroxyphenyl)adamantane (5) was prepared from 4 by treatment with $\mathrm{BBr}_{3}$ in $90 \%$ yield according to the published procedure. ${ }^{19}$ Characterization of product 5 compares well with literature $\left(\mathrm{mp}=301-302{ }^{\circ} \mathrm{C}\right) .{ }^{19} \mathrm{H}$ NMR (DMSO- $d_{6}, 300$ $\mathrm{MHz}) \delta / \mathrm{ppm}: 9.20(\mathrm{~s}, 1 \mathrm{H}, \mathrm{OH}), 7.24(\mathrm{~d}, 2 \mathrm{H}, J=8.6 \mathrm{~Hz}), 6.71(\mathrm{~d}, 2 \mathrm{H}$, $J=8.6 \mathrm{~Hz}), 4.30(\mathrm{~s}, 1 \mathrm{H}, \mathrm{OH}), 2.37$ (br s, 3H), 2.31 (br s, $1 \mathrm{H}), 1.78$ (br $\mathrm{s}, 1 \mathrm{H}), 1.50-1.68(\mathrm{~m}, 9 \mathrm{H})$.

Diphenyl-(2-hydoxyphenyl)methanol (6) was prepared from diphenyl-(2-methoxyphenyl)methanol by treatment with $\mathrm{BBr}_{3}$ in $90 \%$ yield according to the published procedure ${ }^{30}$. Characterization of product 6 compares well with literature $(\mathrm{mp}=$ $\left.148-150{ }^{\circ} \mathrm{C}\right) .{ }^{30}{ }^{1} \mathrm{H}$ NMR $\left(\mathrm{CDCl}_{3}, 300 \mathrm{MHz}\right) \delta / \mathrm{ppm}$ : $8.10(\mathrm{~s}, 1 \mathrm{H}$, $\mathrm{OH}), 6.40-7.40(\mathrm{~m}, 14 \mathrm{H}), 3.70(\mathrm{~s}, 1 \mathrm{H}, \mathrm{OH})$.

4-(3-Methoxyphenyl)homoadamantan-4-ol (7) was prepared in a Grignard reaction from homoadamantan-4-one and 3-bromanisol in $67 \%$ yield according to the published procedure. ${ }^{31}$ Characterization of product 7 compares well with literature $\left(\mathrm{mp}=130-132{ }^{\circ} \mathrm{C}\right) .{ }^{31}{ }^{1} \mathrm{H} \mathrm{NMR}\left(\mathrm{CDCl}_{3}, 300 \mathrm{MHz}\right) \delta / \mathrm{ppm}: 7.25$ $(\mathrm{t}, 1 \mathrm{H}, J=8.0 \mathrm{~Hz}), 7.197 .12(\mathrm{~m}, 2 \mathrm{H}), 6.78(\mathrm{~d}, 1 \mathrm{H}, J=8.0 \mathrm{~Hz}), 3.81$ $(\mathrm{s}, 3 \mathrm{H}), 2.81(\mathrm{dd}, 1 \mathrm{H}, J=3.8,15.0 \mathrm{~Hz}), 2.48(\mathrm{~d}, 1 \mathrm{H}, J=14.0 \mathrm{~Hz})$, $2.22-2.03(\mathrm{~m}, 3 \mathrm{H}), 2.01-1.89(\mathrm{~m}, 4 \mathrm{H}), 1.89-1.68(\mathrm{~m}, 4 \mathrm{H}), 1.60-1.47$ $(\mathrm{m}, 4 \mathrm{H})$.

4-(3-Hydroxyphenyl)homoadamantan-4-ol (8) was prepared from 7 by treatment with sodium thiolate in $90 \%$ yield according to the published procedure. ${ }^{19,31}$ Characterization of product 8 compares well with literature $\left(\mathrm{mp}=162-164{ }^{\circ} \mathrm{C}\right) \cdot{ }^{31}{ }^{1} \mathrm{H}$ NMR (DMSO- $\left.d_{6}, 300 \mathrm{MHz}\right) \delta / \mathrm{ppm}: 9.14(\mathrm{~s}, 1 \mathrm{H}), 7.07(\mathrm{dd}(\mathrm{t}), 1 \mathrm{H}, J=7.8$ $\mathrm{Hz}), 7.00(\mathrm{dd}(\mathrm{t}), 1 \mathrm{H}, J=2.0 \mathrm{~Hz}), 6.96(\mathrm{~d}, 1 \mathrm{H}, J=7.8 \mathrm{~Hz}), 6.56(\mathrm{dd}$, $1 \mathrm{H}, J=2.0,7.8 \mathrm{~Hz}), 4.81(\mathrm{~s}, 1 \mathrm{H}), 2.61-2.50(\mathrm{~m}, 2 \mathrm{H}), 2.13-1.95(\mathrm{~m}$, $2 \mathrm{H}), 1.91-1.65(\mathrm{~m}, 7 \mathrm{H}), 1.59-1.33(\mathrm{~m}, 5 \mathrm{H})$.

exo-4-(3-Hydroxyphenyl)protoadamantan-4-ol (9) was prepared from exo-4-(3-methoxyphenyl)protoadamantan-4-ol by treatment with sodium thiolate in $46 \%$ yield according to the published procedure $\left(\mathrm{mp}=176-178^{\circ} \mathrm{C}\right) .{ }^{19,31}$ Characterization of product 9 compares well with literature. ${ }^{31}{ }^{1} \mathrm{H}$ NMR (DMSO- $d_{6}$, $300 \mathrm{MHz}) \delta / \mathrm{ppm} 9.13(\mathrm{~s}, 1 \mathrm{H}), 7.07(\mathrm{dd}(\mathrm{t}), 1 \mathrm{H}, J=7.8 \mathrm{~Hz})$, 6.91-6.86 (m, 2H), $6.57(\mathrm{dd}, 1 \mathrm{H}, J=1.6,7.6 \mathrm{~Hz}), 4.56(\mathrm{~s}, 1 \mathrm{H}), 2.58$ $(\mathrm{dd}(\mathrm{t}), 1 \mathrm{H}, J=8.2 \mathrm{~Hz}), 2.46-2.36(\mathrm{~m}, 1 \mathrm{H}), 2.33-2.24(\mathrm{~m}, 1 \mathrm{H}), 2.07$ $(\mathrm{bs}, 4 \mathrm{H}), 1.74(\mathrm{~d}, 1 \mathrm{H}, J=13.0 \mathrm{~Hz}), 1.67-1.40(\mathrm{~m}, 3 \mathrm{H}), 1.29(\mathrm{dd}(\mathrm{t})$, $2 \mathrm{H}, J=13.0 \mathrm{~Hz}), 1.19(\mathrm{~d}, 1 \mathrm{H}, J=12.0 \mathrm{~Hz})$.

\subsubsection{Synthesis of 2-Hydroxybiphenyl Derivatives $\mathbf{1 0 - 1 5}$}

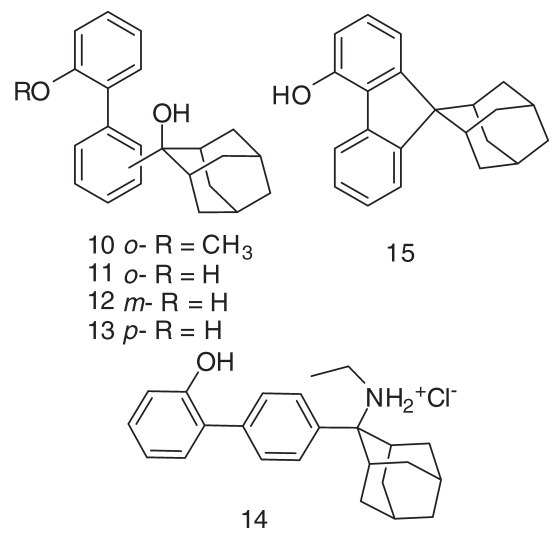

Figure 2 2-Hydoxybiphenyl derivatives. 
2-(2-Hydroxyadamantan-2-yl)-2'-methoxybiphenyl (10) was prepared in a Grignard reaction from adamantan-2-one and 2-bromo-2'-methoxybiphenyl in $76 \%$ yield according to the published procedure. ${ }^{20} \mathrm{Characterization}$ of product 10 compares well with literature $\left(\mathrm{mp}=114-115{ }^{\circ} \mathrm{C}\right) .{ }^{20}{ }^{1} \mathrm{H} \mathrm{NMR}\left(\mathrm{CDCl}_{3}, 600\right.$ $\mathrm{MHz}) \delta / \mathrm{ppm}: 7.68(\mathrm{~d}, 1 \mathrm{H}, J=8.1 \mathrm{~Hz}), 7.38(\mathrm{dt}, 1 \mathrm{H}, J=1.6 \mathrm{~Hz}, J=$ $8.1 \mathrm{~Hz}), 7.34(\mathrm{dt}, 1 \mathrm{H}, J=1.7 \mathrm{~Hz}, J=8.1 \mathrm{~Hz}), 7.27(\mathrm{dt}, 1 \mathrm{H}, J=1.1 \mathrm{~Hz}$, $J=7.4 \mathrm{~Hz}), 7.18(\mathrm{dd}, 1 \mathrm{H}, J=1.6 \mathrm{~Hz}, J=7.4 \mathrm{~Hz}), 7.00-7.04(\mathrm{~m}, 2 \mathrm{H})$, $6.93(\mathrm{~d}, 1 \mathrm{H}, J=8.3 \mathrm{~Hz}), 3.86(\mathrm{br} \mathrm{s}, 1 \mathrm{H}, \mathrm{OH}), 3.74\left(\mathrm{~s}, 3 \mathrm{H}, \mathrm{OCH}_{3}\right) 2.59$ $(\mathrm{br} \mathrm{s}, 1 \mathrm{H}), 2.34(\mathrm{dt}, 1 \mathrm{H}, J=2.2 \mathrm{~Hz}, J=12.4 \mathrm{~Hz}), 2.11(\mathrm{dt}, 1 \mathrm{H}, J=2.2$ $\mathrm{Hz}, J=12.4 \mathrm{~Hz}), 1.80-1.90(\mathrm{~m}, 2 \mathrm{H}), 1.60-1.72(\mathrm{~m}, 5 \mathrm{H}), 1.54(\mathrm{dt}, 1 \mathrm{H}$, $J=2.2 \mathrm{~Hz}, J=12.4 \mathrm{~Hz}), 1.40(\mathrm{dt}, 1 \mathrm{H}, J=2.2 \mathrm{~Hz}, J=12.4 \mathrm{~Hz}), 1.32$ $(\mathrm{dq}, 1 \mathrm{H}, J=3.2 \mathrm{~Hz}, J=12.4 \mathrm{~Hz}), 1.23(\mathrm{dq}, 1 \mathrm{H}, J=3.2 \mathrm{~Hz}, J=12.4$ $\mathrm{Hz})$.

2-(2-Hydroxyadamantan-2-yl)-2'-hydroxybiphenyl (11) was prepared from 10 by treatment with sodium thiolate in $60 \%$ yield according to the published procedure. ${ }^{20}$ Characterization of product 11 compares well with literature $\left(\mathrm{mp}=194-196^{\circ} \mathrm{C}\right) .{ }^{20}$ ${ }^{1} \mathrm{H}$ NMR (DMSO- $\left.d_{6}, 300 \mathrm{MHz}\right), \delta / \mathrm{ppm}: 9.83(\mathrm{~s}, 1 \mathrm{H}, \mathrm{OH}), 7.60(\mathrm{~d}$ $1 \mathrm{H}, J=7.4 \mathrm{~Hz}), 7.36(\mathrm{dt}, 1 \mathrm{H} J=1.5 \mathrm{~Hz}, J=7.3 \mathrm{~Hz}), 7.26(\mathrm{dt}, 1 \mathrm{HJ}=$ $0.9 \mathrm{~Hz}, J=7.3 \mathrm{~Hz}), 7.17(\mathrm{dt}, 1 \mathrm{HJ}=1.5 \mathrm{~Hz}, J=7.5 \mathrm{~Hz}), 7.09(\mathrm{dd}, 1 \mathrm{H}$, $J=1.5 \mathrm{~Hz}, J=7.5 \mathrm{~Hz}), 6.95(\mathrm{dd}, 1 \mathrm{H}, J=1.5 \mathrm{~Hz}, J=7.5 \mathrm{~Hz})$, 6.83-6.90 (m, 2H), 5.30 (s, 1H, OH), 2.50 (br s, 1H), $2.24(\mathrm{~d}, 1 \mathrm{H}$, $J=12.1 \mathrm{~Hz}), 2.08(\mathrm{br} \mathrm{s}, 2 \mathrm{H}), 1.75(\mathrm{~d}, 1 \mathrm{H}, J=11.6 \mathrm{~Hz}), 1.44-1.69(\mathrm{~m}$, $6 \mathrm{H}), 1.36(\mathrm{~d}, 1 \mathrm{H}, J=12.1 \mathrm{~Hz}), 1.29(\mathrm{~d}, 1 \mathrm{H}, J=12.1 \mathrm{~Hz}), 1.21(\mathrm{~d}, 1 \mathrm{H}$, $J=11.6 \mathrm{~Hz})$.

3-(2-Hydroxyadamantan-2-yl)-2'-hydroxybiphenyl (12) was prepared from 3-(2-hydroxyadamantan-2-yl)-2'-methoxybiphenyl by treatment with $\mathrm{BBr}_{3}$ in $69 \%$ yield according to the published procedure. ${ }^{20}$ Characterization of product 12 compares well with literature $\left(\mathrm{mp}=179-181^{\circ} \mathrm{C}\right) \cdot{ }^{20}{ }^{1} \mathrm{H}$ NMR (DMSO- $d, 300$

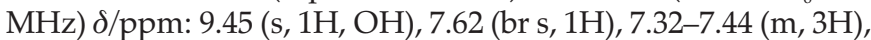
$7.23(\mathrm{dd}, 1 \mathrm{H}, J=1.6 \mathrm{~Hz}, J=7.5 \mathrm{~Hz}), 7.14(\mathrm{dt}, 1 \mathrm{H}, J=1.6 \mathrm{~Hz}, J=8.0$ $\mathrm{Hz}), 6.92(\mathrm{dd}, 1 \mathrm{H}, J=0.8 \mathrm{~Hz}, J=8.0 \mathrm{~Hz}), 6.87(\mathrm{dt}, 1 \mathrm{H}, J=1.0 \mathrm{~Hz}$, $J=7.4 \mathrm{~Hz}), 4.60(\mathrm{br} \mathrm{s}, 1 \mathrm{H}, \mathrm{OH}), 2.50(\mathrm{br} \mathrm{s}, 2 \mathrm{H}), 2.41$ (br s, $1 \mathrm{H}), 2.38$ (br s, 1H), 1.55-1.85 (m, 10H).

4-(2-Hydroxyadamantan-2-yl)-2'-hydroxybiphenyl (13) was prepared from 4-(2-hydroxyadamantan-2-yl)-2'-methoxybiphenyl by treatment with $\mathrm{BBr}_{3}$ in $40 \%$ yield according to the published procedure ${ }^{20}$ Characterization of product 13 compares well with literature $\left(\mathrm{mp}=215-217^{\circ} \mathrm{C}\right) .{ }^{20}{ }^{1} \mathrm{H}$ NMR (DMSO- $d, 300$ $\mathrm{MHz}), \delta /$ ppm: 9.48 (s, 1H, OH), $7.52(\mathrm{~d}, 2 \mathrm{H}, J=8.6 \mathrm{~Hz}), 7.48(\mathrm{~d}$, $2 \mathrm{H}, J=8.6 \mathrm{~Hz}), 7.25(\mathrm{dd}, 1 \mathrm{H} J=1.5 \mathrm{~Hz}, J=7.4 \mathrm{~Hz}), 7.13(\mathrm{dt}, 1 \mathrm{HJ}=$ $1.5 \mathrm{~Hz}, J=8.1 \mathrm{~Hz}), 6.93(\mathrm{dd}, 1 \mathrm{H}, J=1.0 \mathrm{~Hz}, J=8.1 \mathrm{~Hz}), 6.86(\mathrm{dt}, 1 \mathrm{H}$ $J=1.0 \mathrm{~Hz}, J=7.4 \mathrm{~Hz}), 4.57(\mathrm{~s}, 1 \mathrm{H}, \mathrm{OH}), 2.50(\mathrm{br} \mathrm{s}, 1 \mathrm{H}), 2.42(\mathrm{br} \mathrm{s}$, $1 \mathrm{H}), 2.38$ (br s, 1H), 1.82 (br s, 1H), 1.55-1.75 (m, $10 \mathrm{H})$.

4-[2-(N-ethylaminoadamantan-2-yl)]-2'-hydroxybiphenyl hydrochloride (14) was prepared from 4-(2-azidoadamantan-2-yl)2 '-hydroxybiphenyl by reduction with Raney $\mathrm{Ni}$ in $27 \%$ yield according to the published procedure $\left(\mathrm{mp}=242-245^{\circ} \mathrm{C}\right) .{ }^{20} \mathrm{Char}-$ acterization of product $\mathbf{1 4}$ compares well with literature. ${ }^{20}$ ${ }^{1} \mathrm{HNMR}$ (DMSO- $d_{6}, 300 \mathrm{MHz}$ ) $\delta / \mathrm{ppm}: 9.69$ (s, $\left.1 \mathrm{H}, \mathrm{OH}\right), 8.43$ (br s, $\left.2 \mathrm{H}, \mathrm{NH}_{2}\right), 7.69(\mathrm{~d}, 2 \mathrm{H}, J=7.6 \mathrm{~Hz}), 7.65(\mathrm{~d}, 2 \mathrm{H}, J=7.6 \mathrm{~Hz}), 7.31(\mathrm{dd}$, $1 \mathrm{H}, J=7.3 \mathrm{~Hz}, J=1.3 \mathrm{~Hz}), 7.18(\mathrm{dt}, 1 \mathrm{H}, J=8.0 \mathrm{~Hz}, J=1.5 \mathrm{~Hz}), 6.99$ $(\mathrm{d}, 1 \mathrm{H}, J=8.0 \mathrm{~Hz}), 6.89(\mathrm{dd}, 1 \mathrm{H}, J=7.3 \mathrm{~Hz}, J=8.0 \mathrm{~Hz}), 3.35(\mathrm{q}, 2 \mathrm{H}$, $J=7.4 \mathrm{~Hz}), 2.90(\mathrm{br} \mathrm{s}, 2 \mathrm{H}), 2.61(\mathrm{br} \mathrm{s}, 1 \mathrm{H}), 2.47$ (br s, 1H), 2.42 (br s, 1H), 1.94 (br s, $1 \mathrm{H}), 1.62-1.86(\mathrm{~m}, 8 \mathrm{H}), 1.05(\mathrm{t}, 3 \mathrm{H}, J=7.4 \mathrm{~Hz})$.

Spiro[adamantane-2,9'-(4'-hydroxy)fluorene] (15) was prepared from 10 by treatment with $\mathrm{BBr}_{3}$ in $99 \%$ yield according to the published procedure. ${ }^{20}$ Characterization of product 15 compares well with literature $\left(\mathrm{mp}=158-160{ }^{\circ} \mathrm{C}\right) .{ }^{20}{ }^{1} \mathrm{H} \mathrm{NMR}\left(\mathrm{CDCl}_{3}, 600\right.$

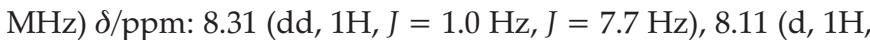
$J=8.1 \mathrm{~Hz}), 7.71(\mathrm{~d}, 1 \mathrm{H}, J=7.9 \mathrm{~Hz}), 7.37(\mathrm{dt}, 1 \mathrm{H}, J=0.8 \mathrm{~Hz}, J=7.6$ $\mathrm{Hz}), 7.26(\mathrm{dt}, 1 \mathrm{H}, J=1.5 \mathrm{~Hz}, J=7.5 \mathrm{~Hz}), 7.12(\mathrm{t}, 1 \mathrm{H}, J=7.9 \mathrm{~Hz})$, $6.72(\mathrm{~d}, 1 \mathrm{H}, J=7.9 \mathrm{~Hz}), 5.08(\mathrm{~s}, 1 \mathrm{H}, \mathrm{OH}), 2.85-3.00(\mathrm{~m}, 4 \mathrm{H}), 2.18(\mathrm{br}$ $\mathrm{s}, 2 \mathrm{H}), 1.98$ (br s, 2H), 1.70-1.80 (m, 4H), 1.55-1.60 (m, 2H).

\subsubsection{Synthesis of 3-Hydroxybiphenyl Derivatives 16-21}

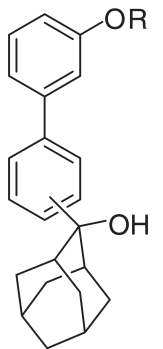

$16 o-\mathrm{R}=\mathrm{H}$ $17 m-\mathrm{R}=\mathrm{CH}_{3}$ $18 m-\mathrm{R}=\mathrm{H}$ $19 p-\mathrm{R}=\mathrm{H}$

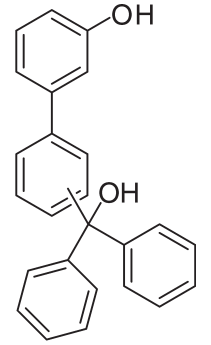

$20 \mathrm{~m}$ $21 p-$
Figure 3 3-Hydoxybiphenyl derivatives.

2-(2-Hydroxyadamantan-2-yl)-3'-hydroxybiphenyl (16) was prepared from 2-(2-hydroxyadamantan-2-yl)-3'-methoxybiphenyl by treatment with sodium thiolate in $20 \%$ yield according to the published procedure. ${ }^{22}$ Characterization of product 16 compares well with literature $\left(\mathrm{mp}=119-120{ }^{\circ} \mathrm{C}\right) .{ }^{22}{ }^{1} \mathrm{H}$ NMR (DMSO- $\left.d_{6}, 600 \mathrm{MHz}\right) \delta / \mathrm{ppm}: 9.26(\mathrm{~s}, 1 \mathrm{H}, \mathrm{OH}), 7.56(\mathrm{~d}, 1 \mathrm{H}, J=7.9$ $\mathrm{Hz}), 7.32(\mathrm{dt}, 1 \mathrm{H}, J=1.4 \mathrm{~Hz}, J=8.1 \mathrm{~Hz}), 7.22(\mathrm{t}, 1 \mathrm{H}, J=7.3 \mathrm{~Hz})$, $7.08(\mathrm{t}, 1 \mathrm{H}, J=7.8 \mathrm{~Hz}), 6.98(\mathrm{dd}, 1 \mathrm{H}, J=1.4 \mathrm{~Hz}, J=7.5 \mathrm{~Hz})$, $6.80-6.86(\mathrm{~m}, 2 \mathrm{H}), 6.67(\mathrm{dd}, 1 \mathrm{H}, J=2.2 \mathrm{~Hz}, J=8.1 \mathrm{~Hz}), 4.68(\mathrm{~s}, 1 \mathrm{H}$, $\mathrm{OH}), 2.24$ (br s, 2H), 2.19 (br s, 1H), 2.17 (br s, 1H), 1.63 (br s, 1H), 1.59 (br s, 1H), 1.40-1.55 (m, 6H), 1.28-1.36 (m, 2H).

3-(2-Hydroxyadamantan-2-yl)-3'-methoxybiphenyl (17) was prepared in a Grignard reaction from 3-bromo-3'-methoxybiphenyl and 2-adamantanone in $86 \%$ yield according to the published procedure. ${ }^{22}$ Characterization of product 17 compares well with literature. ${ }^{22}{ }^{1} \mathrm{H}$ NMR $\left(\mathrm{CDCl}_{3}, 300 \mathrm{MHz}\right) \delta / \mathrm{ppm}$ : 7.74 (s, $1 \mathrm{H}), 7.46-7.56(\mathrm{~m}, 2 \mathrm{H}), 7.43(\mathrm{t}, 1 \mathrm{H}, J=7.5 \mathrm{~Hz}), 7.35(\mathrm{t}, 1 \mathrm{H}, J=7.9$ $\mathrm{Hz}), 7.17(\mathrm{~d}, 1 \mathrm{H}, J=7.7 \mathrm{~Hz}), 7.11(\mathrm{t}, 1 \mathrm{H}, J=2.0 \mathrm{~Hz}), 6.89(\mathrm{dd}, 1 \mathrm{H}$, $J=2.3 \mathrm{~Hz}, J=8.1 \mathrm{~Hz}), 3.86\left(\mathrm{~s}, 3 \mathrm{H}, \mathrm{OCH}_{3}\right), 2.62(\mathrm{br} \mathrm{s}, 1 \mathrm{H}), 2.45(\mathrm{br} \mathrm{s}$, 1H), 2.41 (br s, 1H), 1.92 (br s, 1H), 1.68-1.82 (m, 10H).

3-(2-Hydroxyadamantan-2-yl)-3'-hydroxybiphenyl (18) was prepared from 17 by treatment with $\mathrm{BBr}_{3}$ in $83 \%$ yield according to the published procedure. ${ }^{22}$ Characterization of product 18 compares well with literature $\left(\mathrm{mp}=181-183{ }^{\circ} \mathrm{C}\right) .{ }^{22} \mathrm{H}$ NMR (DMSO- $\left.d_{6}, 300 \mathrm{MHz}\right) \delta /$ ppm: 9.49 (br s, 1H, OH), 7.65 (s, 1H), $7.36-7.50(\mathrm{~m}, 3 \mathrm{H}), 7.25(\mathrm{t}, 1 \mathrm{H}, J=7.8 \mathrm{~Hz}), 7.00-7.08(\mathrm{~m}, 2 \mathrm{H}), 6.76$ $(\mathrm{dd}, 1 \mathrm{H}, J=1.2 \mathrm{~Hz}, J=7.8 \mathrm{~Hz}), 2.50(\mathrm{br} \mathrm{s}, 2 \mathrm{H}), 2.42(\mathrm{br} \mathrm{s}, 1 \mathrm{H}), 2.38$ (br s, 1H), 1.82 (br s, 1H), 1.52-1.76 (m, 10H).

4-(2-Hydroxyadamantan-2-yl)-3'-hydroxybiphenyl (19) was prepared from 4-(2-hydroxyadamantan-2-yl)-3'-methoxybiphenyl by treatment with $\mathrm{BBr}_{3}$ in $34 \%$ yield according to the published procedure. ${ }^{22}$ Characterization of product 19 compares well with literature $\left(\mathrm{mp}=201-203^{\circ} \mathrm{C}\right) .{ }^{22} \mathrm{H}$ NMR (DMSO- $d_{6}, 300$ MHz) $\delta /$ ppm: 9.50 (br s, $1 \mathrm{H}, \mathrm{OH}), 7.56(\mathrm{~d}, 2 \mathrm{H}, J=8.6 \mathrm{~Hz}), 7.53(\mathrm{~d}$, $2 \mathrm{H}, J=8.6 \mathrm{~Hz}), 7.24(\mathrm{t}, 1 \mathrm{H}, J=7.6 \mathrm{~Hz}), 7.06(\mathrm{~d}, 1 \mathrm{H}, J=7.6 \mathrm{~Hz}), 7.02$ $(\mathrm{s}, 1 \mathrm{H}), 6.75(\mathrm{dd}, 1 \mathrm{H}, J=1.2 \mathrm{~Hz}, J=7.9 \mathrm{~Hz}), 2.50(\mathrm{br} \mathrm{s}, 2 \mathrm{H}), 2.41(\mathrm{br}$ $\mathrm{s}, 1 \mathrm{H}), 2.38$ (br s 1H), 1.82 (br s $1 \mathrm{H}), 1.54-1.72(\mathrm{~m}, 10 \mathrm{H})$.

(3'-Hydroxybiphenyl-3-yl)diphenylmethanol (20) was prepared from (3'-methoxybiphenyl-3-yl)diphenylmethanol by 
treatment with $\mathrm{BBr}_{3}$ in $96 \%$ yield according to the published procedure. ${ }^{22}$ Characterization of product 20 compares well with literature $\left(\mathrm{mp}=141-142{ }^{\circ} \mathrm{C}\right) .{ }^{22}{ }^{1} \mathrm{H}$ NMR $\left(\mathrm{DMSO}-d_{6}, 600 \mathrm{MHz}\right)$ $\delta /$ ppm: $9.47(\mathrm{~s}, 1 \mathrm{H}, \mathrm{OH}), 7.50(\mathrm{t}, 1 \mathrm{H}, J=1.4 \mathrm{~Hz}), 7.48(\mathrm{dd}, 1 \mathrm{H}$, $J=1.7 \mathrm{~Hz}, J=7.7 \mathrm{~Hz}), 7.36(\mathrm{t}, 1 \mathrm{H}, J=7.7 \mathrm{~Hz}), 7.32(\mathrm{dd}, 4 \mathrm{H}, J=7.2$ $\mathrm{Hz}, J=7.6 \mathrm{~Hz}), 7.23-7.28(\mathrm{~m}, 6 \mathrm{H}), 7.21(\mathrm{t}, 1 \mathrm{H}, J=7.8 \mathrm{~Hz}), 7.13(\mathrm{dd}$, $1 \mathrm{H}, J=1.5 \mathrm{~Hz}, J=7.7 \mathrm{~Hz}), 6.95(\mathrm{dd}, 1 \mathrm{H}, J=1.3 \mathrm{~Hz}, J=7.7 \mathrm{~Hz})$ $6.92(\mathrm{t}, 1 \mathrm{H}, J=2.0 \mathrm{~Hz}), 6.73(\mathrm{dd}, 1 \mathrm{H}, J=8.0 \mathrm{~Hz}, J=2.3 \mathrm{~Hz}), 6.51(\mathrm{~s}$, $1 \mathrm{H}, \mathrm{OH})$.

(3'-Hydroxybiphenyl-4-yl)diphenylmethanol (21) was prepared from (3'-methoxybiphenyl-4-yl)diphenylmethanol by treatment with $\mathrm{BBr}_{3}$ in $98 \%$ yield according to the published procedure. ${ }^{22}$ Characterization of product 21 compares well with literature $\left(\mathrm{mp}=168-170{ }^{\circ} \mathrm{C}\right) .^{22}{ }^{1} \mathrm{H}$ NMR $\left(\mathrm{DMSO}-d_{6}, 600 \mathrm{MHz}\right)$

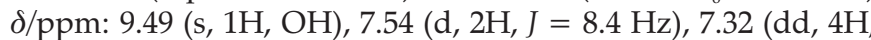
$J=7.2 \mathrm{~Hz}, J=7.7 \mathrm{~Hz}), 7.22-7.30(\mathrm{~m}, 9 \mathrm{H}), 7.06(\mathrm{~d}, 1 \mathrm{H}, J=7.6 \mathrm{~Hz})$, $7.02(\mathrm{t}, 1 \mathrm{H}, J=2.0 \mathrm{~Hz}), 6.75(\mathrm{dd}, 1 \mathrm{H}, J=1.6 \mathrm{~Hz}, J=8.0 \mathrm{~Hz}), 6.46(\mathrm{~s}$, $1 \mathrm{H}, \mathrm{OH})$.

\subsubsection{Synthesis of 4-Hydroxybiphenyl Derivatives 22-31}

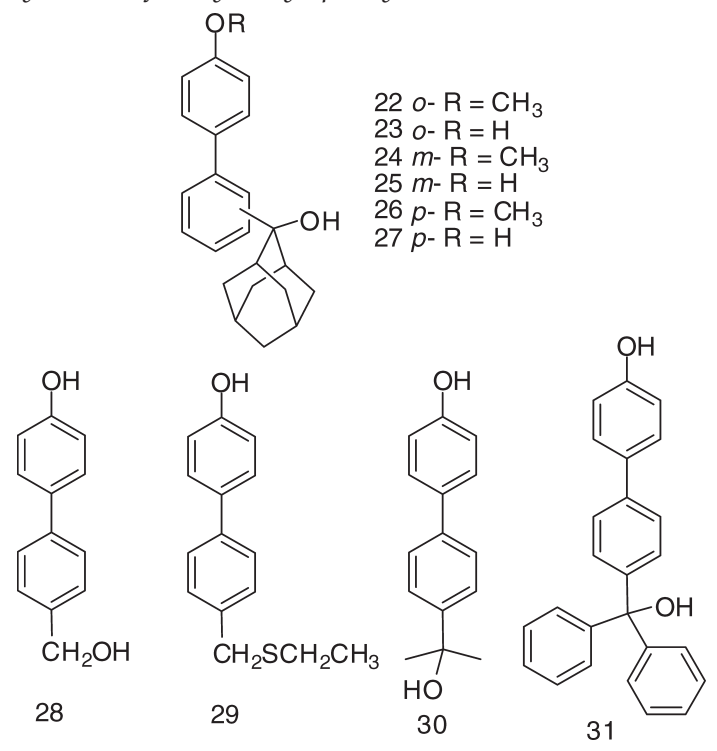

Figure 4 4-Hydoxybiphenyl derivatives.

2-(2-Hydroxyadamantan-2-yl)-4'-methoxybiphenyl (22) was prepared in a Grignard reaction from 2-bromo-4'-methoxybiphenyl and 2-adamantanone in $80 \%$ yield according to the published procedure. ${ }^{21}$ Characterization of product 22 compares well with literature $\left(\mathrm{mp}=125-130{ }^{\circ} \mathrm{C}\right) .{ }^{21}{ }^{1} \mathrm{H}$ NMR $\left(\mathrm{CDCl}_{3}, 300\right.$ $\mathrm{MHz}) \delta / \mathrm{ppm}: 7.61(\mathrm{~d}, 1 \mathrm{H}, J=8.0 \mathrm{~Hz}), 7.32(\mathrm{t}, 1 \mathrm{H}, J=8.0 \mathrm{~Hz}), 7.28$ $(\mathrm{d}, 2 \mathrm{H}, J=8.5 \mathrm{~Hz}), 7.25(\mathrm{t}, 2 \mathrm{H}, J=8.0 \mathrm{~Hz}), 7.10(\mathrm{~d}, 1 \mathrm{H}, J=8.0 \mathrm{~Hz})$, $3.84(\mathrm{~s}, 3 \mathrm{H}), 2.27(\mathrm{~s}, 2 \mathrm{H}), 2.17$ (br s, $1 \mathrm{H}), 2.15(\mathrm{br} \mathrm{s}, 1 \mathrm{H}), 1.81(\mathrm{~s}, 1 \mathrm{H})$, 1.73 (br s, $1 \mathrm{H}), 1.62-1.69(\mathrm{~m}, 3 \mathrm{H}), 1,60$ (br. s, $2 \mathrm{H}), 1.52-1.57(\mathrm{~m}, 2 \mathrm{H})$, $1.49(\mathrm{~d}, 2 \mathrm{H}, J=12.4 \mathrm{~Hz})$.

2-(2-Hydroxyadamantan-2-yl)-4' -hydroxybiphenyl (23) was prepared from 22 by treatment with sodium thiolate in $69 \%$ yield according to the published procedure. ${ }^{21}$ Characterization of product 23 compares well with literature $\left(\mathrm{mp}=160-163^{\circ} \mathrm{C}\right) .^{21}$ ${ }^{1} \mathrm{H}$ NMR (DMSO-d $\left.d_{6}, 300 \mathrm{MHz}\right) \delta / \mathrm{ppm}: 9.29$ (s, 1H, OH), 7.55 (d, $1 \mathrm{H}, J=7.7 \mathrm{~Hz}), 7.29(\mathrm{t}, 1 \mathrm{H}, J=7.7 \mathrm{~Hz}), 7.16-7.26(\mathrm{~m}, 3 \mathrm{H}), 6.98(\mathrm{dd}$, $1 \mathrm{H}, J=1.0 \mathrm{~Hz}, J=7.2 \mathrm{~Hz}), 6.70(\mathrm{~d}, 2 \mathrm{H}, J=8.4 \mathrm{~Hz}), 4.73(\mathrm{~s}, 1 \mathrm{H}$, $\mathrm{OH}), 2.10-2.30(\mathrm{~m}, 4 \mathrm{H}), 1.20-1.70(\mathrm{~m}, 10 \mathrm{H})$.

3-(2-Hydroxyadamantan-2-yl)-4'-methoxybiphenyl (24) was prepared in a Grignard reaction from 3-bromo-4'-methoxybiphenyl and 2-adamantanone in $78 \%$ yield according to the published procedure. ${ }^{21}$ Characterization of product 24 compares well with literature $\left(\mathrm{mp}=103-105^{\circ} \mathrm{C}\right) .{ }^{21}{ }^{1} \mathrm{H} \mathrm{NMR}\left(\mathrm{CDCl}_{3}, 300\right.$ $\mathrm{MHz}), \delta / \mathrm{ppm}: 7.71(\mathrm{br} \mathrm{s}, 1 \mathrm{H}), 7.51(\mathrm{~d}, 2 \mathrm{H}, J=8.8 \mathrm{~Hz}), 7.27-7.49(\mathrm{~m}$, $3 \mathrm{H}), 6.97(\mathrm{~d}, 2 \mathrm{H}, J=8.8 \mathrm{~Hz}), 3.84(\mathrm{~s}, 3 \mathrm{H}), 2.62(\mathrm{br} \mathrm{s}, 2 \mathrm{H}), 2.45(\mathrm{br} \mathrm{s}$, $1 \mathrm{H}), 2.41$ (br s, 1H), 1.91 (br s, 1H), 1.68-1.80 (m, 10H).

3-(2-Hydroxyadamantan-2-yl)-4'-hydroxybiphenyl (25) was prepared from 24 by treatment with $\mathrm{BBr}_{3}$ in $79 \%$ yield according to the published procedure. ${ }^{21}$ Characterization of product 25 compares well with literature $\left(\mathrm{mp}=155-157^{\circ} \mathrm{C}\right) .{ }^{21}{ }^{1} \mathrm{H}$ NMR (DMSO- $d_{6}, 300 \mathrm{MHz}$ ) $\delta / \mathrm{ppm}: 9.50$ (br s, OH), 7.63 (br s, $\left.1 \mathrm{H}\right), 7.46$ $(\mathrm{d}, 2 \mathrm{H}, J=8.6 \mathrm{~Hz}), 7.35-7.44(\mathrm{~m}, 3 \mathrm{H}), 6.85(\mathrm{~d}, 2 \mathrm{H}, J=8.0 \mathrm{~Hz}), 2.52$ (br s, 2H), 2.42 (br s, 1H), 2.38 (br s, 1H), 1,81 (br s, 1H), 1.55-1.70 $(\mathrm{m}, 10 \mathrm{H})$.

4-(2-Hydroxyadamantan-2-yl)-4'-methoxybiphenyl (26) was prepared in a Grignard reaction from 4-bromo-4'-methoxybiphenyl and 2-adamantanone in $65 \%$ yield according to the published procedure ${ }^{21}$ Characterization of product 26 compares well with literature $\left(\mathrm{mp}=186-187^{\circ} \mathrm{C}\right) .{ }^{21}{ }^{1} \mathrm{H}$ NMR $\left(\mathrm{CDCl}_{3}, 300\right.$ $\mathrm{MHz}) \delta /$ ppm: $7.58(\mathrm{~d}, 2 \mathrm{H}, J=8.8 \mathrm{~Hz}), 7.54(\mathrm{~d}, 2 \mathrm{H}, J=8.8 \mathrm{~Hz}), 7.53$ $(\mathrm{d}, 2 \mathrm{H}, J=8.8 \mathrm{~Hz}), 6.97(\mathrm{~d}, 2 \mathrm{H}, J=8.8 \mathrm{~Hz}), 3.84(\mathrm{~s}, 3 \mathrm{H}), 2.59(\mathrm{br} \mathrm{s}$, 2H), 2.45 (br s, 1H), 2.40 (br s, 1H), 1.91 (br s, 1H), 1.70-1.80 (m, $10 \mathrm{H})$.

4-(2-Hydroxyadamantan-2-yl)-4'-hydroxybiphenyl (27) was prepared from 26 by treatment with $\mathrm{BBr}_{3}$ in $67 \%$ yield according to the published procedure. ${ }^{21}$ Characterization of product 27 compares well with literature $\left(\mathrm{mp}=225-228{ }^{\circ} \mathrm{C}\right) .{ }^{21}{ }^{1} \mathrm{H}$ NMR (DMSO- $\left.d_{6}, 300 \mathrm{MHz}\right) \delta / \mathrm{ppm}$ : 9.48 (br s, OH), 7.42-7.61 (m, 6H), $6.84(\mathrm{~d}, 2 \mathrm{H}, \mathrm{J}=8.5 \mathrm{~Hz}), 2.47$ (br s, $2 \mathrm{H}), 2.41$ (br s, $1 \mathrm{H}), 2.38$ (br s, $1 \mathrm{H}), 1.81$ (br s, 1H), 1.53-1.73 (m, 10H).

4-Hydroxymethyl-4'-hydroxybiphenyl (28) was obtained from 4-methoxy-4'-hydroxybiphenyl with sodium thiolate in $23 \%$ yield according to the published procedure..$^{21}$ Characterization of product 28 compares well with literature $\left(\mathrm{mp}=195-196^{\circ} \mathrm{C}\right) .^{21}$ ${ }^{1} \mathrm{H}$ NMR (DMSO- $\left.d_{6}, 300 \mathrm{MHz}\right), \delta / \mathrm{ppm}: 9.50$ (s, 1H, OH), 7.51 (d, $2 \mathrm{H}, J=8.5 \mathrm{~Hz}), 7.46(\mathrm{~d}, 2 \mathrm{H}, J=8.5 \mathrm{~Hz}), 7.33(\mathrm{~d}, 2 \mathrm{H}, J=8.5 \mathrm{~Hz})$, $6.83(\mathrm{~d}, 2 \mathrm{H}, \mathrm{J}=8.6 \mathrm{~Hz}), 5.15(\mathrm{br} \mathrm{s}, 1 \mathrm{H}, \mathrm{OH}), 4.50(\mathrm{~s}, 2 \mathrm{H})$.

4-Thioethylmethyl-4'-hydroxybiphenyl (29) was obtained from 4-methoxy-4'-hydroxybiphenyl with sodium thiolate in $75 \%$ yield according to the published procedure. ${ }^{21}$ Characterization of product 29 compares well with literature. ${ }^{21}{ }^{1} \mathrm{H}$ NMR $\left(\mathrm{CDCl}_{3}, 300 \mathrm{MHz}\right), \delta /$ ppm: $7.48(\mathrm{~d}, 2 \mathrm{H}, \mathrm{J}=8.3 \mathrm{~Hz}), 7.47(\mathrm{~d}, 2 \mathrm{H}$, $J=8.3 \mathrm{~Hz}), 7.36(\mathrm{~d}, 2 \mathrm{H}, J=8.3 \mathrm{~Hz}), 6.89(\mathrm{~d}, 2 \mathrm{H}, J=8.6 \mathrm{~Hz}), 4.84(\mathrm{~s}$, $1 \mathrm{H}, \mathrm{OH}), 3.75(\mathrm{~s}, 2 \mathrm{H}), 2.47(\mathrm{q}, 2 \mathrm{H}, J=7.4 \mathrm{~Hz}), 1.25(\mathrm{t}, 3 \mathrm{H}, J=7.4$ $\mathrm{Hz}) .{ }^{13} \mathrm{C} \mathrm{NMR}\left(\mathrm{CDCl}_{3}, 75 \mathrm{MHz}\right), \delta / \mathrm{ppm}: 155.0$ (s), 139.2 (s), 136.9 $(\mathrm{s}), 133.5(\mathrm{~s}), 129.0(\mathrm{~d}), 128.1(\mathrm{~d}), 126.6(\mathrm{~d}), 115.6(\mathrm{~d}), 35.5(\mathrm{t}), 25.2(\mathrm{t})$, 14.2 (q).

4-Hydroxy-4' -(2-hydroxypropan-2-yl)biphenyl (30) was obtained from 4-methoxy-4'-(2-hydroxypropan-2-yl)biphenyl with sodium thiolate in $80 \%$ yield according to the published procedure. ${ }^{21}$ Characterization of product 30 compares well with literature $\left(\mathrm{mp}=178-180{ }^{\circ} \mathrm{C}\right) .{ }^{21} \mathrm{H}$ NMR (DMSO- $\left.d_{6}, 300 \mathrm{MHz}\right)$, $\delta /$ ppm: $9.48(\mathrm{~s}, 1 \mathrm{H}, \mathrm{OH}), 7.48(\mathrm{~s}, 4 \mathrm{H}), 7.45(\mathrm{~d}, 2 \mathrm{H}, J=8.6 \mathrm{~Hz}), 6.83$ $(\mathrm{d}, 2 \mathrm{H}, J=8.6 \mathrm{~Hz}), 4.98$ (br s, $1 \mathrm{H}, \mathrm{OH}), 1.44(\mathrm{~s}, 6 \mathrm{H})$.

4'-(Hydroxydiphenylmethyl)biphenyl-4-ol (31) was obtained from (4'-methoxybiphenyl-4-yl)diphenylmethanol with $\mathrm{BBr}_{3}$ in $83 \%$ yield according to the published procedure. ${ }^{21}$ Characterization of product 31 compares well with literature $(\mathrm{mp}=$ 274-276 $\left.{ }^{\circ} \mathrm{C}\right) .{ }^{21}{ }^{1} \mathrm{H}$ NMR $\left(\mathrm{CDCl}_{3}, 300 \mathrm{MHz}\right) \delta / \mathrm{ppm}$ : 9.50 (br s $1 \mathrm{H}$, $\mathrm{OH}), 7.49(\mathrm{t}, 4 \mathrm{H}, J=8.7 \mathrm{~Hz}), 7.18-7.34(\mathrm{~m}, 12 \mathrm{H}), 6.83(\mathrm{~d}, 2 \mathrm{H}, J=8.6$ $\mathrm{Hz}), 6.41$ (br s, 1H, OH). 
2.1.5. Synthesis of Naphthols $\mathbf{3 2 - 4 0}$ and the Corresponding QM Derivatives 41 and 42

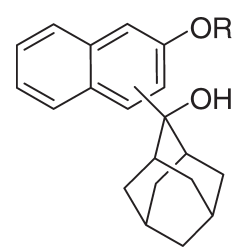

32 2,3- $\mathrm{R}=\mathrm{CH}_{3}$ $332,3-\mathrm{R}=\mathrm{H}$ 342,6- $\mathrm{R}=\mathrm{CH}_{3}$ 352,6- $\mathrm{R}=\mathrm{H}$ $362,7-\mathrm{R}=\mathrm{H}$<smiles>[R]c1cccc2ccccc12</smiles><smiles>NC1(c2ccc3cc(O)ccc3c2)C2CC3CC(C2)CC1C3</smiles>

37
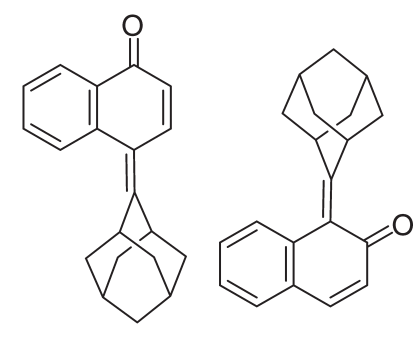

41

42

381,4- $\mathrm{R}=\mathrm{CH}_{3}$ $391,4-\mathrm{R}=\mathrm{CH}_{2}$ Phe 401,5- $\mathrm{R}=\mathrm{H}$

Figure 5 Naphthols and the corresponding QMs.

2-(2-Hydroxy-2-adamantyl)-3-methoxynaphthalene (32) was prepared in a Grignard reaction from 2-bromo-3-methoxynaphthalene and 2-adamantanone in $87 \%$ yield according to the published procedure. ${ }^{23}$ Characterization of product 32 compares well with literature $\left(\mathrm{mp}=208-210{ }^{\circ} \mathrm{C}\right) \cdot{ }^{23} \mathrm{H}$ NMR $\left(\mathrm{CDCl}_{3}, 300\right.$ MHz) $\delta /$ ppm: $7.86(\mathrm{~s}, 1 \mathrm{H}), 7.76(\mathrm{~d}, 1 \mathrm{H}, J=8.0 \mathrm{~Hz}), 7.69(\mathrm{~d}, 1 \mathrm{H}$, $J=8.0 \mathrm{~Hz}), 7.42(\mathrm{dt}, 1 \mathrm{H}, J=1.2 \mathrm{~Hz}, J=8.0 \mathrm{~Hz}), 7.34(\mathrm{dt}, 1 \mathrm{H}, J=1.2$ $\mathrm{Hz}, J=8.0 \mathrm{~Hz}), 7.17(\mathrm{~s}, 1 \mathrm{H}), 3.97\left(\mathrm{~s}, 3 \mathrm{H}, \mathrm{OCH}_{3}\right), 3.56(\mathrm{~s}, 1 \mathrm{H}, \mathrm{OH})$, 2.78 (br s, 2H), 2.55 (br s, 2H), 1.60-2.08 (m, 10H).

2-(2-Hydroxy-2-adamantyl)-3-hydroxynaphthalene (33) was obtained from 32 with $\mathrm{BBr}_{3}$ in $64 \%$ yield according to the published procedure. ${ }^{23}$ Characterization of product 33 compares well with literature $\left(\mathrm{mp}=226-228^{\circ} \mathrm{C}\right){ }^{23}{ }^{1} \mathrm{H}$ NMR (DMSO- $d_{6}, 600$ $\mathrm{MHz}) \delta / \mathrm{ppm}$ : 9.69 (s, 1H, OH), 7.85 (s, $1 \mathrm{H}), 7.80$ (d, 1H, J = 8.1 $\mathrm{Hz}), 7.63(\mathrm{~d}, 1 \mathrm{H}, J=8.1 \mathrm{~Hz}), 7.36(\mathrm{t}, 1 \mathrm{H}, J=8.1 \mathrm{~Hz}), 7.25(\mathrm{t}, 1 \mathrm{H}$, $J=8.1 \mathrm{~Hz}), 7.16(\mathrm{~s}, 1 \mathrm{H}), 5.07(\mathrm{~s}, 1 \mathrm{H}, \mathrm{OH}), 2.76(\mathrm{br} \mathrm{s}, 2 \mathrm{H}), 2.50(\mathrm{br} \mathrm{s}$ $1 \mathrm{H}$, covered by DMSO), 2.47 (br s, $1 \mathrm{H}), 1.80-1.85(\mathrm{~m}, 3 \mathrm{H})$, $1.72-1.80(\mathrm{~m}, 3 \mathrm{H}), 1.67$ (br s, $2 \mathrm{H}), 1.57$ (d, $2 \mathrm{H}, J=11.6 \mathrm{~Hz})$.

2-(2-Hydroxy-2-adamantyl)-6-methoxynaphthalene (34) was prepared in a Grignard reaction from 2-bromo-6-methoxynaphthalene and 2-adamantanone in $81 \%$ yield according to the published procedure. ${ }^{23}$ Characterization of product 34 compares well with literature $\left(\mathrm{mp}=162-163{ }^{\circ} \mathrm{C}\right) .{ }^{23}{ }^{1} \mathrm{H}$ NMR $\left(\mathrm{CDCl}_{3}, 300\right.$ $\mathrm{MHz}) \delta /$ ppm: $7.86(\mathrm{~d}, 1 \mathrm{H}, J=1.1 \mathrm{~Hz}), 7.68-7.76(\mathrm{~m}, 2 \mathrm{H}), 7.63(\mathrm{dd}$, $1 \mathrm{H}, J=1.8 \mathrm{~Hz}, J=8.8 \mathrm{~Hz}), 7.09-7.16(\mathrm{~m}, 2 \mathrm{H}), 3.90\left(\mathrm{~s}, 3 \mathrm{H}, \mathrm{OCH}_{3}\right)$, 2.66 (br s, 2H), 2.47 (br s, 1H), 2.43 (br s, $1 \mathrm{H}), 1.92$ (br s, $1 \mathrm{H})$, $1.68-1.80(\mathrm{~m}, 10 \mathrm{H})$.

2-(2-Hydroxy-2-adamantyl)-6-hydroxynaphthalene (35) was obtained from 34 with $\mathrm{BBr}_{3}$ in $62 \%$ yield according to the published procedure. ${ }^{23} \mathrm{Characterization}$ of product 35 compares well with literature $\left(\mathrm{mp}=196-198^{\circ} \mathrm{C}\right) .{ }^{23}{ }^{1} \mathrm{H}$ NMR (DMSO- $d_{6}, 300$ $\mathrm{MHz}) \delta /$ ppm: 9.63 (s, 1H, OH), 7.80 (br s, 1H), $7.74(\mathrm{~d}, 1 \mathrm{H}, J=8.6$ $\mathrm{Hz}), 7.63(\mathrm{~d}, 1 \mathrm{H}, J=8.8 \mathrm{~Hz}), 7.53(\mathrm{dd}, 1 \mathrm{H}, J=1.2 \mathrm{~Hz}, J=8.8 \mathrm{~Hz})$, 7.01-7.08 (m, 2H), 4.57 (s, 1H, OH), 2.57 (br s, 2H), 2.44 (br s, 1H), 2.40 (br s, 1H), 1.82 (br s, 1H), 1.45-1.65 (m, 9H).

2-(2-Hydroxy-2-adamantyl)-7-hydroxynaphthalene (36) was obtained from 2-(2-hydroxy-2-adamantyl)-7-methoxynaphtha- lene with $\mathrm{BBr}_{3}$ in $63 \%$ yield according to the published procedure. ${ }^{23}$ Characterization of product 36 compares well with literature $\left(\mathrm{mp}=250-252^{\circ} \mathrm{C}\right) .^{23}{ }^{1} \mathrm{H}$ NMR $\left(\mathrm{DMSO}-d_{6}, 300 \mathrm{MHz}\right) \delta / \mathrm{ppm}$ : $7.66-7.74(\mathrm{~m}, 3 \mathrm{H}), 7.40(\mathrm{~d}, 1 \mathrm{H}, J=8.8 \mathrm{~Hz}), 7.11(\mathrm{~d}, 1 \mathrm{H}, J=2.2 \mathrm{~Hz})$, $7.03(\mathrm{dd}, 1 \mathrm{H}, J=2.2 \mathrm{~Hz}, J=8.7 \mathrm{~Hz}), 2.58(\mathrm{br} \mathrm{s}, 2 \mathrm{H}), 2.44(\mathrm{br} \mathrm{s}, 1 \mathrm{H})$, 2.40 (br s, 1H), 1.82 (br s, 1H), 1.56-1.72 (m, 9H).

2-(2-Amino-2-adamantyl)-6-hydroxynaphthalene hydrochloride (37) was obtained from 2-(2-azido-2-adamantyl)-6hydroxynaphthalene by reduction with Raney Ni in $19 \%$ yield according to the published procedure. ${ }^{23}$ Characterization of product 37 compares well with literature $\left(\mathrm{mp}=256-258^{\circ} \mathrm{C}\right) .^{23}$ ${ }^{1} \mathrm{H}$ NMR (DMSO- $d_{6}, 300 \mathrm{MHz}$ ) $\delta / \mathrm{ppm}: 9.92$ (s, 1H), 8.12 (br s, 3H), 7.98 (br s, 1H), 7.75-7.85 (m, 2H), 7.56-7.62 (m, 1H), 7.11-7.16 (m, $2 \mathrm{H}), 2.86$ (br s, 2H), 2.34 (br s, 1H), 2.29 (br s, 1H), 1.63-1.97 (m, $10 \mathrm{H})$.

1-(2-Hydroxy-2-adamantyl)-4-methoxynaphthalene (38) was prepared in a Grignard reaction from 1-bromo-4-methoxynaphthalene and 2-adamantanone in $68 \%$ yield according to the published procedure. ${ }^{32}$ Characterization of product 38 compares well with literature $\left(\mathrm{mp}=211-212{ }^{\circ} \mathrm{C}\right) \cdot{ }^{32} \mathrm{H} \mathrm{NMR}\left(\mathrm{CDCl}_{3}, 300\right.$ $\left.\mathrm{MHz}, 20^{\circ} \mathrm{C}\right) \delta / \mathrm{ppm} 8.78(\mathrm{~d}, 1 \mathrm{H}, J=7.8 \mathrm{~Hz}), 8.31(\mathrm{~d}, 1 \mathrm{H}, J=7.8 \mathrm{~Hz})$, $7.55(\mathrm{~d}, 1 \mathrm{H}, J=8.2 \mathrm{~Hz}), 7.40-7.48(\mathrm{~m}, 2 \mathrm{H}), 6.70(\mathrm{~d}, 1 \mathrm{H}, J=8.2 \mathrm{~Hz})$, $3.99(\mathrm{~s}, 3 \mathrm{H}), 2.64(\mathrm{~d}, 2 \mathrm{H}, J=12.0 \mathrm{~Hz}), 1.93(\mathrm{br} \mathrm{s}, 1 \mathrm{H}), 1.84(\mathrm{br} \mathrm{s}, 1 \mathrm{H})$, $1.68-1.80(\mathrm{~m}, 6 \mathrm{H})$.

1-Benzyloxy-4-(2-hydroxy-2-adamantyl)naphthalene (39) was prepared in a Grignard reaction from 1-benzyloxy-4-bromonaphthalene and 2-adamantanone in $58 \%$ yield according to the published procedure. ${ }^{23}$ Characterization of product 39 compares well with literature $\left(\mathrm{mp}=152-153{ }^{\circ} \mathrm{C}\right) .{ }^{23}{ }^{1} \mathrm{H}$ NMR $\left(\mathrm{CDCl}_{3}, 600\right.$ $\mathrm{MHz}) \delta / \mathrm{ppm}:$ 8.78-8.81 (m, 1H), 8.39-8.48 (m, 1H), 7.52-7.57 (m, $3 \mathrm{H}$, with a discernible doublet at 7.55, $J=8.3 \mathrm{~Hz}), 7.41-7.49(\mathrm{~m}$, $4 \mathrm{H}), 7.35-7.38(\mathrm{~m}, 1 \mathrm{H}), 6.79(\mathrm{~d}, 1 \mathrm{H}, J=8.3 \mathrm{~Hz}), 5.26(\mathrm{~s}, 2 \mathrm{H}), 2.67(\mathrm{br}$ s, $1 \mathrm{H}), 2.65$ (br s, $1 \mathrm{H}), 1.95$ (br s, $1 \mathrm{H}), 1.85(\mathrm{~s}, 1 \mathrm{H}), 1.70-1.82(\mathrm{~m}, 6 \mathrm{H})$.

1-Hydroxy-5-(2-hydroxy-2-adamantyl)naphthalene (40) was prepared by catalytic hydrogenation of 1-benzyloxy-5-(2-hydroxy-2-adamantyl)naphthalene in $82 \%$ yield according to the published procedure. ${ }^{23}$ Characterization of product 40 compares well with literature $\left(\mathrm{mp}=218-220{ }^{\circ} \mathrm{C}\right) \cdot{ }^{23}{ }^{1} \mathrm{H}$ NMR $\left(\mathrm{CD}_{3} \mathrm{OD}\right)$ $\delta /$ ppm: $8.26(\mathrm{~d}, 1 \mathrm{H}, J=8.8 \mathrm{~Hz}), 8.17(\mathrm{~d}, 1 \mathrm{H}, J=8.5 \mathrm{~Hz}), 7.65(\mathrm{~d}, 1 \mathrm{H}$, $J=7.4 \mathrm{~Hz}), 7.32(\mathrm{dd}, 1 \mathrm{H}, J=8.2, J=7.5 \mathrm{~Hz}), 7.16(\mathrm{dd}, 1 \mathrm{H}, J=8.8$, $J=7.5 \mathrm{~Hz}), 6.75(\mathrm{~d}, 1 \mathrm{H}, J=7.5 \mathrm{~Hz}), 2.70(\mathrm{~s}, 1 \mathrm{H}), 2.69(\mathrm{~s}, 1 \mathrm{H}), 1.87$ (br s,1H), 1.62-1.81 (m, 6H);

1-(2-Adamantylidene)naphthalene-2(1H)-one (41) was obtained in a reaction of 1-bromo-2-hydroxynaphthalene with BuLi and 2-adamantanone in $48 \%$ yield according to the published procedure. ${ }^{23}$ Characterization of product 41 compares well with literature $\left(\mathrm{mp}=152-153^{\circ} \mathrm{C}\right) .^{23}{ }^{1} \mathrm{H}$ NMR (DMSO- $d_{6}, 600$ MHz) $\delta$ /ppm: 7.48-7.52 (m, 2H), 7.30-7.37 (m, 3H), 6.22 (d, 1H, $J=9.8 \mathrm{~Hz}), 4.10$ (br s, $1 \mathrm{H}), 3.43$ (br s, $1 \mathrm{H}), 1.94-2.02(\mathrm{~m}, 6 \mathrm{H})$, $1.84-1.90(\mathrm{~m}, 4 \mathrm{H}), 1.77(\mathrm{~d}, 2 \mathrm{H}, J=12.2 \mathrm{~Hz})$.

4-(2-Adamantylidene)naphthalene-1(4H)-one (42) was obtained by hydrogenation of 39 in $83 \%$ yield according to the published procedure. ${ }^{23}$ Characterization of product 42 compares well with literature $\left(\mathrm{mp}=158-160^{\circ} \mathrm{C}\right){ }^{23}{ }^{1} \mathrm{H}$ NMR (DMSO- $d_{6}, 600$ MHz) $\delta / p p m: ~ 8.14(\mathrm{~d}, 1 \mathrm{H}, J=10.3 \mathrm{~Hz}), 8.04(\mathrm{dd}, 1 \mathrm{H}, J=7.8 \mathrm{~Hz}$, $J=1.4 \mathrm{~Hz}), 7.71(\mathrm{~d}, 1 \mathrm{H}, J=7.8 \mathrm{~Hz}), 7.64(\mathrm{dt}, 1 \mathrm{H}, J=1.3 \mathrm{~Hz}, J=8.0$ $\mathrm{Hz}), 7.51(\mathrm{dt}, 1 \mathrm{H}, J=0.9 \mathrm{~Hz}, J=7.4 \mathrm{~Hz}), 6.3(\mathrm{~d}, 1 \mathrm{H}, J=10.3 \mathrm{~Hz})$, $3.73(\mathrm{~s}, 1 \mathrm{H}), 3.61(\mathrm{~s}, 1 \mathrm{H}), 1.852 .17(\mathrm{~m}, 12 \mathrm{H})$.

\subsection{Antimicrobial Testing}

Preliminary antimicrobial screening of the compounds was 
determined in duplicate using a modification of the Kirby-Bauer disc diffusion method. All compounds were dissolved in dimethyl sulfoxide (DMSO) and tested against Staphylococcus aureus ATCC 25923 (Gram-positive), Bacillus subtilis ATCC 6633 (Gram-positive), Candida albicans ATCC 10231 (fungus) Escherichia coli ATCC 25922 (Gram-negative) and Klebsiella pneumonia ATCC 31488 (Gram-negative). The microbial cultures were grown overnight at $37^{\circ} \mathrm{C}$ on Nutrient Agar plates (Biolab, South Africa), adjusted to $0.5 \mathrm{McF}$ arlands standard using distilled water and lawn inoculated onto Mueller-Hinton agar (MHA) plates (Biolab, South Africa). $30 \mu \mathrm{L}$ of each sample was inoculated onto antibiotic assay discs (6 $\mathrm{mm}$ diameter) and placed on the MHA plates which were incubated overnight at $37^{\circ} \mathrm{C}$ and zones of inhibition were measured. DMSO was used as a control. Thereafter, minimum inhibitory concentrations (MIC) were determined in triplicate with compounds displaying antimicrobial activity using the broth dilution method. Serial dilutions (10000-19.56 $\mu \mathrm{g} / \mathrm{mL}$ ) of the compounds (with the exception of compounds $16[4200-8.20 \mu \mathrm{g} / \mathrm{mL}$ ) and 37 [4700-9.18 $\mu \mathrm{g} / \mathrm{mL}]$ ) were prepared from the stock solutions and tested against the cultures used in the preliminary antimicrobial activity studies.

\section{Results and Discussion}

MICs as determined by the broth dilution method are presented in Table 1. Generally, the compounds are not active against Gram-negative bacteria. The only exception was compound 37 that exhibited very weak activity against $E$. coli. Similarly, only compounds 3 and 41 exhibited antifungal activity, but only at higher concentrations. On the contrary, compounds were found to be more potent against Gram-positive bacteria. This finding suggests that the antimicrobial activity is not due to the non-specific ability to alter membranes, but rather an unknown mechanism of action. The activity against Gram-positive bacteria has been reported also for the adamantyl derivatives of oxadiazoles, thioureas and thiadiazoles. ${ }^{25,26,29}$ However, the activity data are not comparable since different activity tests were employed..$^{33}$ Adamantyl derivative of Platensimycin was found also as extremely potent against Gram-positive methicillinresistant Staphylococcus aureus (MRSA) exhibiting activity in the range $1-2 \mu \mathrm{g} / \mathrm{mL}^{27,28}$

With respect to the SAR it is difficult to make a straightforward conclusion, since compounds from each library were found to be active. However, the findings of this study suggest that only compounds with two aromatic rings exert activity against
Gram-positive bacteria. Both 2-hydroxy-2-adamantyl and diphenylmethanol derivatives were found to be active against the tested strains, suggesting that incorporation of the adamantyl group does not enhance the activity. With respect to the substitution on the biphenyl skeleton, better activities were observed for derivatives of 3- and 4-, than for 2-hydroxybiphenyl. From the naphthalene derivatives only compound 37 was found to be active. The data suggest that the ammonium functional group at the benzylic position (compounds 14 and 37) instead of the hydroxyl (compounds $\mathbf{1 3}$ and 35) probably contributes to the enhancement of the antimicrobial effect. Since the ammonium is a better leaving group, this finding suggests that the activity could be correlated with the ability of these compounds to be metabolically transformed to QMs. The most active compound is QM 41. However, the structure of QM is also important since the other QM derivative 42 exerts no activity. To verify the proposed action mechanism of the investigated compounds it would be of significant importance to detect QMs formed in a metabolic process inside the living cells. However, such a detection is not warranted since all QMs except 41 and 42 are transient species, reactive intermediates with submillisecond lifetimes. ${ }^{19-24}$

\section{Conclusion}

Five groups of compounds containing in total 42 phenol and naphthol derivatives were synthesized and screened for antimicrobial activity. Generally, compounds were found to be more active against Gram-positive bacteria suggesting some new mechanism of action. From the SAR studies it can be concluded that the mode of action can be correlated with the ability of compounds to from QMs. This hypothesis is to be further tested, and if shown to be true, will have an impact in the further design of antimicrobial agents.

\section{Acknowledgements}

These materials are based on work financed by the Croatian Foundation for Science (HRZZ grant no. 02.05/25 and IP-201409-6312), the Ministry of Science Education and Sports of the Republic of Croatia (grant No. 098-0982933-2911), The National Research Foundation (South Africa), Aspen Pharmacare and the University of KwaZulu-Natal.

\section{Supplementary Material}

The online supplement contains a table with zones of inhibition (in $\mathrm{mm}$ ) of the compounds $(n=3)$.

Table 1 MIC $(\mu \mathrm{g} / \mathrm{mL})$ of the compounds ${ }^{\text {a }}(n=3)$.

\begin{tabular}{|c|c|c|c|c|c|c|c|c|}
\hline Compound & $\begin{array}{c}\text { Staphylococcus aureus } \\
\text { ATCC } 25923\end{array}$ & SD & $\begin{array}{c}\text { Bacillus subtilis } \\
\text { ATCC } 6633\end{array}$ & SD & $\begin{array}{l}\text { Candida albicans } \\
\text { ATCC } 10231\end{array}$ & SD & $\begin{array}{c}\text { Escherichia coli } \\
\text { ATCC } 25922\end{array}$ & $\mathrm{SD}$ \\
\hline 3 & - & & - & & 5000 & 2 & - & \\
\hline 14 & 2500 & 2 & 625 & 1 & - & & - & \\
\hline 16 & 525 & 1 & 131.2 & 0.1 & - & & - & \\
\hline 20 & 625 & 0 & 625 & 0.1 & - & & - & \\
\hline 21 & 5000 & 2 & 413.67 & 1.8 & - & & - & \\
\hline 23 & 312.5 & 1 & 625 & 0.1 & - & & - & \\
\hline 25 & 5000 & 2 & - & & - & & - & \\
\hline 31 & 520.83 & 180.42 & 416.67 & 1.82 & - & & - & \\
\hline 37 & 1175 & 1 & 587.5 & 1 & - & & 2350 & 1 \\
\hline 41 & 76.1 & 0.1 & 76.1 & 0.1 & 2500 & 1 & - & \\
\hline
\end{tabular}

${ }^{a}$ The MIC is defined as the minimum drug concentration that inhibits growth of the organism and is represented as an average. The MICs were determined in triplicate, using the broth dilution method. The compounds were dissolved in DMSO and DMSO was used as a control.

(-) indicates no compound activity.

SD- standard deviation. 
I. Žabčić, K. Mlinarić-Majerski and N. Basarić,

S. Afr. J. Chem., 2016, 69, 44-50,

$<$ http://journals.sabinet.co.za/sajchem/>.

\section{References}

1 J.J. Lucchini, J. Corre and A. Cremieux, Antibacterial activity of phenolic compounds and aromatic alcohols. Res. Microb., 1990, 141, 499-510.

2 C. Cueva, M.V. Moreno-Arribas, P. Martín-Álvarez, G. Bills, M.F. Vicente, A. Basilio, C. López Rivas, T. Requena, J. M. Rodríguez and B. Bartolomé, Antimicrobial activity of phenolic acids against commensal, probiotic and phatogenic bacteria. Res. Microb., 2010, 161, 372-382.

3 W.B. Hugo, Phenols: a review of their history and development as antimicrobial agents. Microbios., 1978, 23, 83-85.

4 M. Lang and R.M. Rye, The uptake by E. coli and growth inhibitory properties of benzyl alcohol and phenethyl alcohol. J. Pharm. Pharmacol., 1972, 24, 219-226.

5 O'Neal and T.M. Buttle, Effects of alcohols on micro-organisms. Adv. Microb. Physiol., 1984, 25, 253-300.

6 J. Corre, J.J. Lucchini, G.M. Mercier and A. Cremieux, Antibacterial activity of phenethyl alcohol and resulting membrane alterations. Res. Microb., 1990, 141, 483-497.

7 S. Silver and L. Wendt, Mechanism of action of phenethyl alcohol: breakdown of cellular permeability barrier. J. Bact. 1967, 93, 560-566.

8 M.T. Silva, J.C.F. Sousa, M.A.E. Macedo, J. Polonia and A.M. Parente, Effects of phenethyl alcohol on Bacillus and Streptococcus. J. Bact., 1976, 127, 1359-1369.

9 P. Gilbert, E.G. Beveridge and P.B. Corne, Effect of phenoxyethanol on the permeability of E. coli NCTC 5933 to inorganic ions. Microbios., 1977, 19, 17-26.

10 R.G. Kroll and G.D. Anagnostopoulos, Potassium fluxes on hyperosmotic shock and the effect of phenol and bronopol (2-bromo-3nitropropan-1,3-diol) on deplasmolysis of P. aeruginosa. J. Appl. Bact., 1981, 51, 313-323.

11 M.L. Higgins, T.J. Shaw, M.C. Tillman and F.R. Leach, Effect of phenethyl alcohol on cell culture growth. Exp. Cell Res., 1969, 56 24-28.

12 L. Jurd, A.D. King Jr., K. Mihara and W.L. Stanley, Antimicrobial properties of natural phenols and related compounds. Appl Microb., 1971, 21, 507-510.

13 L.J. Nohynek, H.-L. Alakomi, M.P. Kähkönen, M. Heinonen, I.M. Helander, K.-M. Oksman-Caldentey and R.H. Puupponen-Pimiä, Berry phenolics: antimicrobial properties and mechanisms of action against severe human pathogens. Nutrition Cancer., 2006, 54, 18-32.

14 C. Papadopoulou, K. Soulti and I.G. Roussis, Potential antimicrobial activity of red and white wine phenolic extracts against strains of Staphylococcus aureus, Escherichia coli and Candida albicans. Food Technol. Biotechnol., 2005, 43, 41-46.

15 S.O. Salawu, A.O. Ogundare, B.B. Ola-Salawu and A.A. Akindahunsi, Antimicrobial activities of phenolic containing extracts of some tropical vegetables. African J. Pharm. Pharmacol., 2011, 5, 486-492.

16 M.J. Alves, I.C.F.R. Ferreira, H.J.C. Froufe, R.M.V. Abreu, A. Martins and M. Pintado, Antimicrobial activity of phenolic compounds identified in wild mushrooms, SAR analysis and docking studies. J. Appl. Microb., 2013, 115, 346-357.

17 Y. Shen, M.H. Hwang, S. Roffler and C.F. Chen, Cytotoxicity and antimicrobial activity of some naphthol derivatives. Arch. Pharm., 1995, 328, 197-201.

18 M. Reboud-Ravaux and M. Wakselman, Quinone methides and aza-quinone methides as latent alkylating species in the design of mechanism-based inhibitors of serine protease and $\beta$-lactamase, in
S. Rokita, Quinone Methides, John Wiley and Sons, Hoboken, USA, 2009, p 357-383.

19 N. Basarić, I. Žabčić, K. Mlinarić-Majerski and P. Wan, Photochemical formation and chemistry of long-lived adamantylidene quinone methides and 2-adamantyl cations. J. Org. Chem., 2010, 75, 102-116.

20 N. Basarić, N. Cindro, Y. Hou, I. Žabčić, K. Mlinarić-Majerski and P. Wan, Competing photodehydration and excited state intramolecular proton transfer (ESIPT) in adamantyl derivatives of 2-phenylphenols, Can. J. Chem., 2011, 89, 221-234.

21 N. Basarić, N. Cindro, D. Bobinac, Mlinarić-Majerski, L. Uzelac, M. Kralj and P. Wan, Sterically congested quinone methides in photodehydration reactions of 4-hydroxybiphenyl derivatives and investigation of their antiproliferative activity. Photochem. Photobiol. Sci. 2011, 10, 1910-1925.

22 N. Basarić, N. Cindro, D. Bobinac, L. Uzelac, K. Mlinarić-Majerski, M. Kralj and P. Wan, Zwitterionic biphenyl quinone methides in photodehydration reactions of 3-hydroxybiphenyl derivatives: laser flash photolysis and antiproliferation study, Photochem. Photobiol. Sci., 2012, 11, 381-396.

23 J. Veljković, L. Uzelac, K. Molčanov, K. Mlinarić-Majerski, M. Kralj, P. Wan and N. Basarić, Sterically congested adamantylnaphthalene quinone methides. J. Org. Chem., 2012, 77, 4596-4610.

24 Đ. Škalamera, K. Mlinarić-Majerski, I. Martin-Kleiner, M. Kralj and P. Wan, Near-visible light generation of a quinone methide from 3-hydroxymethyl-2-anthrol, J. Org. Chem., 2014, 79, 4390-4397.

25 R.E. Lee, M. Protopopova, E. Crooks, R.A. Slayden, M. Terrot and C.E. Barry, Combinatorial lead optimization of [1,2]-diamines based on ethambutol as potential antituberculosis preclinical candidates. J. Comb. Chem., 2003, 5, 172-187.

26 M. Protopopova, C. Hanrahan, B. Nikonenko, R. Samala, P. Chen, J. Gearhart, L. Einck and C.A. Nacy, Identification of a new antitubercular drug candidate, SQ109, from a combinatorial library of 1,2ethylenediamines. J. Antimicrob. Chemother., 2005, 56, 968-974.

27 K.C. Nicolaou, T. Lister, R.M. Denton, A. Montero and D.J. Edmons, Adamantaplatensimycin: a bioactive analogue of platensimycin. Angew. Chem. Int. Ed., 2007, 46, 4712-4714.

28 K.C. Nicolaou, A.F. Stepan, T. Lister, A. Li, A. Montero, G. Scott Tria, C.I. Turner, Y. Tang, J. Wang, R.M. Denton and D.J. Edmons, Design, synthesis, and biological evaluation of platensimycin analogues with varying degrees of molecular complexity. J. Am. Chem. Soc., 2008, 130, 13110-13119.

29 L. Wanka, K. Iqbal and P.R. Schreiner, The lipophilic bullet hits the targets: medicinal chemistry of adamantane derivatives. Chem Rev., 2013, 113, 3516-3604.

30 L. Diao and P. Wan, Chemistry of photogenerated $\alpha$-phenyl-substituted $o_{-}, m_{-}$, and $p$-quinone methides from phenol derivatives in aqueous solution. Can. J. Chem., 2008, 86, 105-118.

31 N. Cindro, I. Antol, K. Mlinarić-Majerski, I. Halasz, P. Wan and N. Basarić, Reactivity of cations and zwitterions formed in photochemical and acid-catalyzed reactions from $m$-hydroxycycloalkylsubstituted phenol derivatives. J. Org. Chem., 2015, 80, 12420-12430.

32 J. Veljković, I. Antol, N. Basarić, V. Smrečki, K. Molčanov, N. Müller and K. Mlinarić-Majerski, Atropisomerism in 1-(2-adamantyl)naphthalene derivatives, J. Mol. Struct. 2013, 1046, 101-109.

33 A.A. Kadi, N.R. El-Brollosy, O.A. Al-Deeb, E.E. Habib, T.M. Ibrahim and A.A. El-Amam, Synthesis, antimicrobial, and anti-inflammatory activities of novel 2-(1-adamantyl)-5-substituted-1,3,4-oxadiazoles and 2-(1-adamantylamino)-5-substituted-1,3,4-thiadiazoles. Eur. J. Med. Chem., 2007, 42, 235-242 


\section{Supplementary Information, S. Afr. J. Chem.}

In vitro investigation of the antimicrobial activity of a series of lipophilic phenols and naphthols

Thavendran Govender, ${ }^{\mathrm{a}}$ Usha Govinden, ${ }^{\mathrm{a}}$ Chunderika Mocktar, ${ }^{\mathrm{a}}$ Hendrik G. Kruger, ${ }^{\mathrm{a}}$ Jelena

Veljković, ${ }^{\mathrm{b}}$ Nikola Cindro, ${ }^{\mathrm{b}}$ Damir Bobinac, ${ }^{\mathrm{b}}$ Ivana Žabčić, ${ }^{\mathrm{b}}$ Kata Mlinarić-Majerski, ${ }^{\mathrm{b}}$ Nikola Basarić $^{b *}$

${ }^{a}$ Catalysis and Peptide Research Unit, School of Health Sciences, University of KwaZuluNatal, Durban, South Africa. Fax: +27 31 2603091;

${ }^{b}$ Department of Organic Chemistry and Biochemistry, Ruđer Bošković Institute, Bijenička cesta 54, 10000 Zagreb, Croatia. Fax: +385 14680 195; E-mail: nbasaric@irb.hr

\section{TABLE OF CONTENTS}

1. Zones of inhibition of compounds (Table S1) 
Table S1: Zones of inhibition (in $\mathrm{mm}$ ) of the compounds $(\mathrm{n}=3$ )

\begin{tabular}{|c|c|c|c|c|c|c|c|c|}
\hline Compound & $\begin{array}{l}\text { Staphylococcus } \\
\text { aureus } \\
\text { ATCC } 25923\end{array}$ & SD & $\begin{array}{l}\text { Bacillus } \\
\text { subtilis } \\
\text { ATCC } \\
6633\end{array}$ & SD & $\begin{array}{l}\text { Candida } \\
\text { albicans } \\
\text { ATCC } \\
10231\end{array}$ & SD & $\begin{array}{l}\text { Escherichia } \\
\text { coli } \\
\text { ATCC } \\
25922\end{array}$ & SD \\
\hline 3 & - & & - & & 8.83 & 0.29 & - & \\
\hline 14 & 14.33 & 0.6 & 24.33 & 0.6 & - & & - & \\
\hline 16 & 13.33 & 0.5 & 17.67 & 0.6 & - & & - & \\
\hline 20 & 14.67 & 0.6 & 15.33 & 0.5 & - & & - & \\
\hline 21 & 10.33 & 0.6 & 13.33 & 0.6 & - & & - & \\
\hline 23 & 17.33 & 0.6 & 16.33 & 0.6 & - & & - & \\
\hline 25 & 9 & 0.1 & - & & - & & - & \\
\hline 31 & 13.67 & 0.6 & 13.67 & 0.5 & - & & - & \\
\hline 37 & 13.67 & 0.5 & 18.33 & 0.6 & - & & 10.33 & 0.6 \\
\hline 41 & 20.33 & 0.6 & 19.67 & 0.6 & 11.67 & 0.6 & - & \\
\hline
\end{tabular}

Compounds were dissolved in DMSO and DMSO was used as a control.

(-) indicates no compound activity 


\section{Supplementary Information, S. Afr. J. Chem.}

In vitro investigation of the antimicrobial activity of a series of lipophilic phenols and naphthols

Thavendran Govender, ${ }^{\mathrm{a}}$ Usha Govinden, ${ }^{\mathrm{a}}$ Chunderika Mocktar, ${ }^{\mathrm{a}}$ Hendrik G. Kruger, ${ }^{\mathrm{a}}$ Jelena

Veljković, ${ }^{\mathrm{b}}$ Nikola Cindro, ${ }^{\mathrm{b}}$ Damir Bobinac, ${ }^{\mathrm{b}}$ Ivana Žabčić, ${ }^{\mathrm{b}}$ Kata Mlinarić-Majerski, ${ }^{\mathrm{b}}$ Nikola Basarić $^{b *}$

${ }^{a}$ Catalysis and Peptide Research Unit, School of Health Sciences, University of KwaZuluNatal, Durban, South Africa. Fax: +27 31 2603091;

${ }^{b}$ Department of Organic Chemistry and Biochemistry, Ruđer Bošković Institute, Bijenička cesta 54, 10000 Zagreb, Croatia. Fax: +385 14680 195; E-mail: nbasaric@irb.hr

\section{TABLE OF CONTENTS}

1. Zones of inhibition of compounds (Table S1) 
Table S1: Zones of inhibition (in $\mathrm{mm}$ ) of the compounds $(\mathrm{n}=3$ )

\begin{tabular}{|c|c|c|c|c|c|c|c|c|}
\hline Compound & $\begin{array}{l}\text { Staphylococcus } \\
\text { aureus } \\
\text { ATCC } 25923\end{array}$ & SD & $\begin{array}{l}\text { Bacillus } \\
\text { subtilis } \\
\text { ATCC } \\
6633\end{array}$ & SD & $\begin{array}{l}\text { Candida } \\
\text { albicans } \\
\text { ATCC } \\
10231\end{array}$ & SD & $\begin{array}{l}\text { Escherichia } \\
\text { coli } \\
\text { ATCC } \\
25922\end{array}$ & SD \\
\hline 3 & - & & - & & 8.83 & 0.29 & - & \\
\hline 14 & 14.33 & 0.6 & 24.33 & 0.6 & - & & - & \\
\hline 16 & 13.33 & 0.5 & 17.67 & 0.6 & - & & - & \\
\hline 20 & 14.67 & 0.6 & 15.33 & 0.5 & - & & - & \\
\hline 21 & 10.33 & 0.6 & 13.33 & 0.6 & - & & - & \\
\hline 23 & 17.33 & 0.6 & 16.33 & 0.6 & - & & - & \\
\hline 25 & 9 & 0.1 & - & & - & & - & \\
\hline 31 & 13.67 & 0.6 & 13.67 & 0.5 & - & & - & \\
\hline 37 & 13.67 & 0.5 & 18.33 & 0.6 & - & & 10.33 & 0.6 \\
\hline 41 & 20.33 & 0.6 & 19.67 & 0.6 & 11.67 & 0.6 & - & \\
\hline
\end{tabular}

Compounds were dissolved in DMSO and DMSO was used as a control.

(-) indicates no compound activity 


\section{Supplementary Information, S. Afr. J. Chem.}

In vitro investigation of the antimicrobial activity of a series of lipophilic phenols and naphthols

Thavendran Govender, ${ }^{\mathrm{a}}$ Usha Govinden, ${ }^{\mathrm{a}}$ Chunderika Mocktar, ${ }^{\mathrm{a}}$ Hendrik G. Kruger, ${ }^{\mathrm{a}}$ Jelena

Veljković, ${ }^{\mathrm{b}}$ Nikola Cindro, ${ }^{\mathrm{b}}$ Damir Bobinac, ${ }^{\mathrm{b}}$ Ivana Žabčić, ${ }^{\mathrm{b}}$ Kata Mlinarić-Majerski, ${ }^{\mathrm{b}}$ Nikola Basarić $^{b *}$

${ }^{a}$ Catalysis and Peptide Research Unit, School of Health Sciences, University of KwaZuluNatal, Durban, South Africa. Fax: +27 31 2603091;

${ }^{b}$ Department of Organic Chemistry and Biochemistry, Ruđer Bošković Institute, Bijenička cesta 54, 10000 Zagreb, Croatia. Fax: +385 14680 195; E-mail: nbasaric@irb.hr

\section{TABLE OF CONTENTS}

1. Zones of inhibition of compounds (Table S1) 
Table S1: Zones of inhibition (in $\mathrm{mm}$ ) of the compounds $(\mathrm{n}=3$ )

\begin{tabular}{|c|c|c|c|c|c|c|c|c|}
\hline Compound & $\begin{array}{l}\text { Staphylococcus } \\
\text { aureus } \\
\text { ATCC } 25923\end{array}$ & SD & $\begin{array}{l}\text { Bacillus } \\
\text { subtilis } \\
\text { ATCC } \\
6633\end{array}$ & SD & $\begin{array}{l}\text { Candida } \\
\text { albicans } \\
\text { ATCC } \\
10231\end{array}$ & SD & $\begin{array}{l}\text { Escherichia } \\
\text { coli } \\
\text { ATCC } \\
25922\end{array}$ & SD \\
\hline 3 & - & & - & & 8.83 & 0.29 & - & \\
\hline 14 & 14.33 & 0.6 & 24.33 & 0.6 & - & & - & \\
\hline 16 & 13.33 & 0.5 & 17.67 & 0.6 & - & & - & \\
\hline 20 & 14.67 & 0.6 & 15.33 & 0.5 & - & & - & \\
\hline 21 & 10.33 & 0.6 & 13.33 & 0.6 & - & & - & \\
\hline 23 & 17.33 & 0.6 & 16.33 & 0.6 & - & & - & \\
\hline 25 & 9 & 0.1 & - & & - & & - & \\
\hline 31 & 13.67 & 0.6 & 13.67 & 0.5 & - & & - & \\
\hline 37 & 13.67 & 0.5 & 18.33 & 0.6 & - & & 10.33 & 0.6 \\
\hline 41 & 20.33 & 0.6 & 19.67 & 0.6 & 11.67 & 0.6 & - & \\
\hline
\end{tabular}

Compounds were dissolved in DMSO and DMSO was used as a control.

(-) indicates no compound activity 